\title{
CALCULATE WAVELETS SPECTRAL ESTIMATE
}

\author{
Natallia Semianchuk \\ Faculty of Mathematics and Computer Science, Grodno State University, Grodno, Belarus \\ se_nata@tut.by
}

\begin{abstract}
The main task of this research work is applying techniques of wavelet analysis in spectral analysis of stationary random processes. The algorithm of calculation obtained for the spectral estimate of stationary random processes with discrete time via Doubeshies scaling function is studied.
\end{abstract}

\section{Introduction}

One of the main problems in spectral analysis of time series is consistent estimate formation of the second order spectral density via finite realization of stationary process. In numerous research dealing with the mentioned task periodogram methods based on inverse Fourier transform are used.

It's essential to mention that various research questions for statistics of consistent estimate obtained by periodogram smoothing via spectral windows are published, for example, in monographs [1-3] and publications [4-10].

Recent application of wavelet-analysis methods in time series study is quite relevant, as the results obtained from this method are frequently more informative and can directly deal with such input data peculiarities which are difficult to handle with the traditional approach.

Instead of using a deterministic approach scientists usually use a stochastic approach to model the data and to estimate the energy distribution (e.g. in electrical engineering, geophysics, economics or neurophysiology). One reason is that in a stochastic setup certain fluctuations of the Fourier-transform of the data can be interpreted more naturally.

\section{Scaling Doubeshies function}

Consider scaling Doubeshies function $\varphi(x) \in L_{2}(\mathbf{R})$ of order $L \in \mathbf{N}$. The scaling function $\varphi(x)$ is continuous and has the following properties (see [4]):

$$
\int_{\mathbf{R}} \varphi(x) d x=1,
$$




$$
\varphi(x)=\sqrt{2} \sum_{k \in Z} h_{k} \varphi(2 x-k), \quad x \in \mathbf{R}, \operatorname{supp} \varphi(x)=[0 ; 2 L-1],
$$

where $h_{k}, k \in \mathbf{Z}$ - filter of scaling function, $\operatorname{supp} \varphi(x)$ - support of scaling function.

Table 1

Example of Doubeshies scaling function (order $L=2, L=3$ )

\begin{tabular}{|c|c|}
\hline \multicolumn{2}{|c|}{$L=2$} \\
\hline$h_{0}$ & 0.48296291314453410 \\
\hline$h_{1}$ & 0.836516337378077 \\
\hline$h_{2}$ & 0.2241438680420134 \\
\hline$h_{3}$ & -0.1294095225512603 \\
\hline \multicolumn{2}{|c|}{$L=3$} \\
\hline$h_{0}$ & 0.3326705529500825 \\
\hline$h_{1}$ & 0.8068915093110924 \\
\hline$h_{2}$ & 0.4598775021184914 \\
\hline$h_{3}$ & -0.1350110200102546 \\
\hline$h_{4}$ & -0.0854412738820267 \\
\hline$h_{5}$ & 0.0352262918857095 \\
\hline
\end{tabular}

On Figures 1 and 2 some examples of the scaling Doubeshies function with compact support are illustrated.

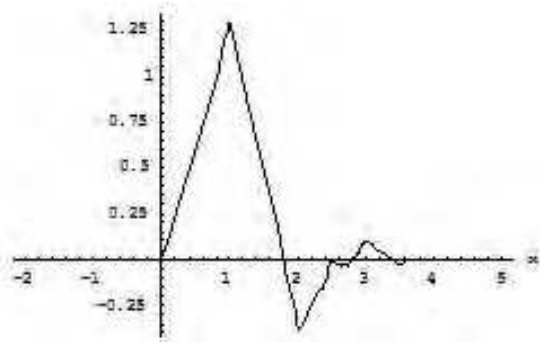

Fig. 1. Scaling Doubeshies function $L=3$, $\max _{x \in \mathbf{R}}|\varphi(x)|=1.28634$ $\operatorname{supp} \varphi(x) \subset[0,5]$

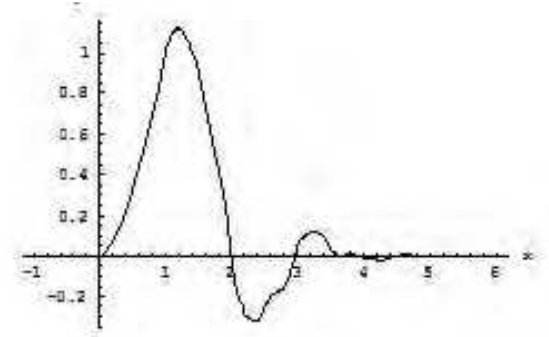

Fig. 2. Scaling Doubeshies function $L=4, \max _{x \in \mathbf{R}}|\varphi(x)|=1.12165$ $\operatorname{supp} \varphi(x) \subset[0,7]$

We can construct $2 \pi$-periodical scaling, using scaling Doubeshies function via formula (1): 


$$
\widetilde{\varphi}_{j, k}(\lambda)=\frac{2^{\frac{j}{2}}}{\sqrt{2 \pi}} \sum_{n=-1}^{(2 L-1) 2^{-j}+1} \varphi\left(\frac{2^{j} \lambda}{2 \pi}+2^{j} n-k\right), \lambda \in \Pi .
$$

Function (1) is formed orthonormal basis of spase $L_{2}(\Pi)$. trated.

On Figure 3 some examples of the $2 \pi$-periodical scaling function are illus-

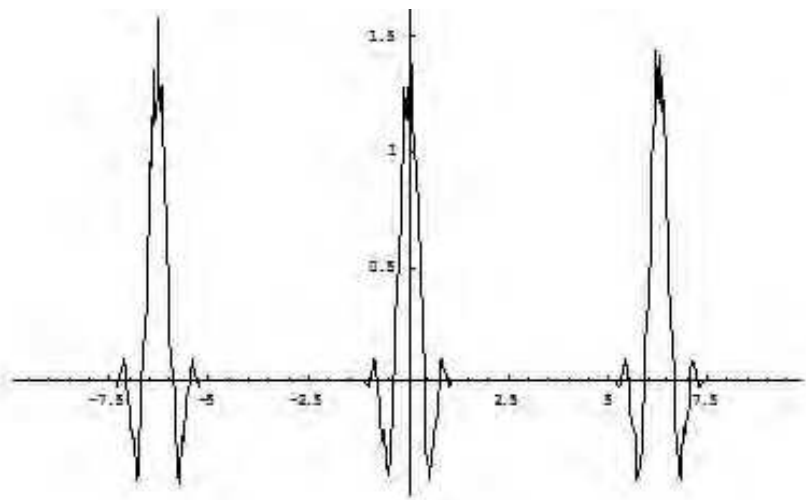

Fig. 3. $2 \pi$-periodical scaling function

\section{Spectral estimate}

Let $X(t), t \in \mathbf{Z}$, be a wide-sense stationary stochastic process with $E X(t)=0$, $t \in \mathbf{Z}$, belonging to a set of random processes $\chi\left(\lambda, f, \alpha, L, C_{2}\right)$.

The set $\chi\left(\lambda, f, \alpha, L, C_{2}\right)$ is defined as the set of wide-sense stationary processes $\mathrm{X}(t), t \in \mathbf{Z}$, whose spectral density is $f(\lambda), \lambda \in \Pi=[-\pi, \pi]$, having a fourthorder semi-invariant spectral density $f_{4}\left(\lambda_{1}, \lambda_{2}, \lambda_{3}\right), \lambda_{j} \in \Pi, j=\overline{1,3}$, and such that for fixed $\lambda \in \Pi$ the spectral density $f$ satisfies $f \in \operatorname{Lip}_{\alpha}(L)$ and the fourthorder semi-invariant spectral density is bounded by a constant $C_{2}>0$.

The definition of the class $\chi\left(\lambda, f, \alpha, L, C_{2}\right)$ can be found in Zhurbenko [9]. It contains processes with spectral densities whose peaks and troughs increase with $T$, for example $A R$-process with peaks [5].

For a process $X \in \chi\left(\lambda, f, \alpha, L, C_{2}\right)$, the rate of convergence of the mean-square deviation of a linear wavelet estimate of the spectral density is studied in [6].

The coefficients of the asymptotically dominant term, which depend on the smoothness of the spectral density, are calculated for some scaling functions and data tapering windows you can find in [5]. 
Thus the information on value $\alpha$ according to the aprioristic information on spectral density for investigated stochastic process. Such information, as a rule, is undertaken on the basis of supervision over several realizations for the concrete phenomenon.

As spectral estimate $f(\lambda)$ let's consider statistics:

$$
\hat{f}(\lambda)=\sum_{k=1}^{2^{J}} \hat{\alpha}_{J, k} \widetilde{\varphi}_{J, k}(\lambda)
$$

where

$$
\hat{\alpha}_{J, k}=\int_{\Pi} I_{T}(\alpha) \widetilde{\varphi}_{J, k}(\alpha) d \alpha
$$

are wavelet-coefficients estimates in (2), and $I_{T}(\lambda)$ - modified periodogram:

$$
\begin{gathered}
I_{T}(\lambda)=\frac{1}{2 \pi H_{2}^{(T)}(0)} d_{T}(\lambda) d_{T}(-\lambda) \\
d_{T}(\lambda)=\sum_{t=0}^{T-1} h_{T}(t) X(t) e^{-i \lambda t} \\
H_{k}^{(T)}(\lambda)=\sum_{t=0}^{T-1}\left(h_{T}(t)\right)^{k} e^{-i \lambda t}
\end{gathered}
$$

$k \in \mathbf{N}, T \in \mathbf{N}$, and function $h_{T}(t)=h\left(\frac{t}{T}\right), h:[0,1] \rightarrow \mathbf{R}$ - data taper, its behavior is studied sufficiently in $[2,6]$.

$$
\widetilde{\varphi}_{J, k}(\lambda)=\sum_{n \in Z}(2 \pi)^{-1 / 2} \varphi_{J, k}\left((2 \pi)^{-1} \lambda+n\right)
$$

- $2 \pi$ - periodical scaling function;

$$
\varphi_{J, k}(x)=2^{J / 2} \varphi\left(2^{J} x-k\right),
$$

$J \in \mathbf{N}_{0}=\{0,1,2, \ldots\}, \lambda \in \Pi, \varphi(x)$ - scaling function $x \in \mathbf{R}$. We have to mention that the results cited in this article are obtained with condition of data tapers variation restriction.

In the spectral analysis we can use the following data taper:

1. Function $h(x), x \in[-1,1]$, bounded variation, $h(x)=1$ in point $x=0$, and $h(x)=0$ for $|x| \geq 1$, and $h(x)=0$ for $x<0$. 
2. Function $h(x)$, bounded variation, with $h(x)=1$ in point $x=\frac{1}{2}$, and $h(x)=0$ for $x \leq 0$ and $x \geq 1$.

A heuristic explanation is the following. Straightforward calculation gives for the expectation of the wavelet estimation:

$$
E \hat{f}(\lambda)=\iint_{\Pi^{2}} f(\lambda+x+\gamma) \Phi_{2}^{(T)}(\gamma) K_{J}(\lambda, \lambda+x) d x d \gamma
$$

where

$$
\begin{aligned}
K_{J}(\lambda, \alpha) & =\sum_{k=1}^{2^{J}} \widetilde{\varphi}_{J, k}(\lambda) \widetilde{\varphi}_{J, k}(\alpha) \\
\Phi_{2}^{(T)}(x) & =\frac{H_{1}^{(T)}(x) H_{1}^{(T)}(-x)}{2 \pi H_{2}^{(T)}(0)}
\end{aligned}
$$

are two kernel functions. One is dependent on the scaling function, the second on data taper.

In paper [6] it is proved that for first moment's estimate (2) we have:

$$
E \hat{f}(\lambda)=f(\lambda)+O\left(\frac{1}{2^{J \alpha}}\right)+R_{T},
$$

where

$$
R_{T}=\left\{\begin{array}{l}
O\left(\frac{1}{T^{\alpha}}\right), \text { if } 0<\alpha<1 \\
O\left(\frac{\ln (\pi T)}{T}\right), \text { if } \alpha=1
\end{array}\right.
$$

In fact, one can prove that

$$
\hat{E f}(\lambda)=f(\lambda)+O\left(T^{-2}\right)
$$

for a sufficiently smooth data taper and for a scaling function of higher order. For dispersion it is valid:

$$
\begin{aligned}
D(\hat{f}(\lambda))= & \frac{2 \pi H_{4}^{(T)}(0)}{\left(H_{2}^{(T)}(0)\right)^{2}} \sum_{k_{1}=1}^{2^{J}} \sum_{k_{2}=1}^{2^{J}} \widetilde{\varphi}_{J, k_{1}}(\lambda) \widetilde{\varphi}_{J, k_{2}}(\lambda) \int_{\Pi} \widetilde{\varphi}_{J, k_{1}}(\alpha)\left(\widetilde{\varphi}_{J, k_{2}}(-\alpha)+\widetilde{\varphi}_{J, k_{2}}(\alpha)\right) f^{2}(\alpha) d \alpha+ \\
& +O\left(\frac{1}{T}\right)+O\left(\frac{2^{J} \ln ^{5}(\pi T)}{T^{1+\alpha}}\right)+O\left(\frac{2^{J(\mu+1)} \ln ^{5}(\pi T)}{T^{1+\mu}}\right)+O\left(\frac{2^{2 J} \ln ^{3}(\pi T)}{T^{2}}\right)
\end{aligned}
$$


Wavelet estimate $\hat{f}(\lambda)$, defined by formula (1), is consistent in the meansquare sense estimate of spectral density $f(\lambda), \lambda \in \Pi$ for all $2^{J} \leq R T^{1-\rho}$, where $0<\rho<1,0<R<\infty$, is some fixed constant.

\section{Calculation}

Step 1. Choose data taper $h_{T}(t)$. Data taper $h_{T}(t)=h\left(\frac{t}{T}\right)$ can be found from condition of minimization of value:

$$
\Delta=\int_{-\pi}^{\pi}|x|^{\alpha}\left|\Phi_{2}^{(T)}(x)\right| d x+\frac{2}{T} \sum_{k=0}^{\left[\frac{T}{2}\right]-1}\left(\left|\frac{H_{2}^{(T)}\left(\frac{2 \pi k}{T}\right)}{H_{2}^{(T)}(0)}\right|\right)^{2} \rightarrow \min _{H}
$$

where $H$ - same bounded set of data taper $h(x), x \in \mathbf{R}$.

The integral

$$
\int_{-\pi}^{\pi}|x|^{\alpha}\left|\Phi_{2}^{(T)}(x)\right| d x
$$

and the sum

$$
\frac{2}{T} \sum_{k=0}^{\left[\frac{T}{2}\right]-1}\left(\left|\frac{H_{2}^{(T)}\left(\frac{2 \pi k}{T}\right)}{H_{2}^{(T)}(0)}\right|\right)^{2}
$$

can be calculated, using standard numerical methods. More examples of data taper functions are given below:

1. Hemming's window:

$$
h(x)=0.54+0.46 \cos (\pi x), x \in[-1,1] .
$$

2. A triangular window:

$$
h(x)=1-|x|, x \in[-1,1] .
$$

3. Rice's, Bohner's, Parzen's window:

$$
h(x)=1-x^{2}, x \in[-1,1] .
$$


The data taper, chosen via this method is optimal in the sense of minimum mean-square deviation of the modified periodogram.

Thus, the advantages of data tapers could also be established theoretically. The choice of the data taper is an important problem, for which no rigorous results exist. It is obvious that the choice depends on the true (unknown) spectral density, in particular on the relation of the peaks and troughs to each other.

Step 2. Calculate modified periodogram $I_{T}(\lambda)$, via formulas (3) and (4), using data taper $h(x), x \in[-1,1]$, which we find on step 1 .

Step 3. Choose scaling function

$$
\left(\frac{(2 \pi)^{\alpha} 2 L \max _{\varphi}|\varphi(x)|}{T^{\alpha}}\left[\int_{\mathbf{R}}|z|^{\alpha}|\varphi(z)| d z\right]\right)^{2} \rightarrow \min _{\Phi}
$$

where $\Phi=\left\{\varphi_{L}(x), x \in \mathbf{R}, L \in \mathbf{N}\right\}$ - the set of Doubeshies scaling function of different order.

The choice of scaling function is influenced by two characteristics by: maximum of scaling function and its support.

Step 4. Calculate level $J$

$$
J=\left[\log _{2}(T)\right],
$$

where [•] - the whole part of number.

Step 5. For calculation of initial factors we will put $J_{0}=T$.

Step 6. Coefficients $\hat{\alpha}_{J_{0}, k}$ calculate, using formula of left rectangle (see step 6.1), or using quadrature formula (see step 6.2).

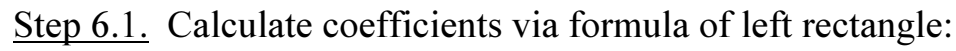

$$
\hat{\alpha}_{J_{0}, k}=\sqrt{2^{-J_{0}+1} \pi} \frac{(2 L-1)}{N_{1}} \sum_{l=0}^{N_{1}-1} I_{T}\left(2^{-J_{0}+1} \pi\left(k+\frac{2 L-1}{N_{1}} l\right)\right) \varphi\left(\frac{2 L-1}{N_{1}} l\right),
$$

where: $N_{1}$ - quantity of parts for integration interval $\left(N_{1} \leq T\right), k=\overline{0,2^{J_{0}}-1}$.

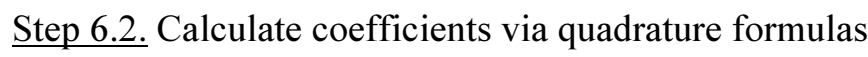

$$
\hat{\alpha}_{J_{0}, k} \approx 2^{\frac{-J_{0}+1}{2}} \sqrt{\pi} \sum_{k=1}^{r} \omega_{k} I_{T}\left(\frac{\left(x_{k}+k\right)}{2^{J_{0}-1}}\right),
$$


$k=\overline{0,2^{J_{0}}-1}$; where method of are calculated of abscissas $x_{k} k=\overline{1, r}$ and weight $\omega_{k}, k=\overline{1, r}$ is considered in paper [7].

Table 2

Example of abscissas $x_{k}, k=\overline{1, r}$ and weight $\omega_{k}, k=\overline{1, r}$, of quadrature formulas for Doubeshies system of order $L=2, L=3$

\begin{tabular}{|c|c|c|c|}
\hline & $k$ & $x_{k}$ & $\omega_{k}$ \\
\hline \multirow{3}{*}{$\begin{array}{l}L=2 \\
r=3\end{array}$} & 1 & 0.565179 & 0.899173 \\
\hline & 2 & 1.565179 & 0.132858 \\
\hline & 3 & 2.565179 & -0.032031 \\
\hline \multirow{4}{*}{$\begin{array}{l}L=2 \\
r=4\end{array}$} & 1 & 0.247825 & 0.268749 \\
\hline & 2 & 0.747825 & 0.561228 \\
\hline & 3 & 1.247825 & 0.298997 \\
\hline & 4 & 1.747825 & -0.128975 \\
\hline \multirow{5}{*}{$\begin{array}{l}L=2 \\
r=5\end{array}$} & 1 & 0.253425 & 0.276273 \\
\hline & 2 & 0.753425 & 0.557197 \\
\hline & 3 & 1.253425 & 0.296560 \\
\hline & 4 & 1.753425 & -0.130903 \\
\hline & 5 & 2.253425 & 0.000872 \\
\hline \multirow{3}{*}{$\begin{array}{l}L=3 \\
r=3\end{array}$} & 1 & 0.804695 & 0.990491 \\
\hline & 2 & 2.804695 & 0.012666 \\
\hline & 3 & 4.804695 & -0.003156 \\
\hline \multirow{4}{*}{$\begin{array}{l}L=3 \\
r=4\end{array}$} & 1 & 0.701350 & 0.817228 \\
\hline & 2 & 1.701350 & 0.264924 \\
\hline & 3 & 2.701350 & -0.097581 \\
\hline & 4 & 3.701350 & 0.015430 \\
\hline \multirow{5}{*}{$\begin{array}{l}L=3 \\
r=5\end{array}$} & 1 & 0.661075 & 0.747720 \\
\hline & 2 & 1.661075 & 0.384507 \\
\hline & 3 & 2.661075 & -0.174764 \\
\hline & 4 & 3.661075 & 0.048801 \\
\hline & 5 & 4.661075 & -0.006264 \\
\hline
\end{tabular}

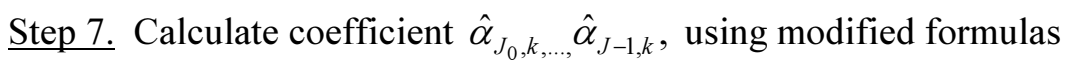

$$
\hat{\alpha}_{j, k}=\frac{1}{L} \sum_{n=0}^{2 L-1} h_{n} \hat{\alpha}_{j+1,(n+2 k) \bmod 2^{j+1}},
$$

where $h_{k}, k \in \mathbf{Z}$ - filters of Doubeshies scaling function. 
Step 8. Build wavelet estimate $\hat{f}(\lambda)$, using formula

$$
\hat{f}(\lambda)=\sum_{k=1}^{2^{J}} \hat{\alpha}_{J, k} \widetilde{\varphi}_{J, k}(\lambda)
$$

on level $J$.

Estimate $\hat{f}(\lambda)$, constructed via the considered algorithm is optimal in minimum biases square. We note that other existing spectral estimates have similar resolution properties as the tapered periodogram. Those statistics are usually non-linear, non-quadratic and therefore very difficult to investigate theoretically.

\section{Conclusions}

Our theoretical results in this paper are also used for developing computational algorithms for wavelet estimates of the spectral density. These algorithms enable us:

1) to select a data tapering windows;

2) to choose a scaling function;

3) to compute the level of decomposition;

4) in order to construct the estimate minimizing the mean square deviation, depending on the sample length and the smoothness of the spectral density.

\section{References}

[1] Anderson T., Statistic Analysis of the Time Series, Moscow, Mir 1976.

[2] Brillinger D., Time Series. Data Processing and Theory, Moscow, Mir 1980.

[3] Troush N.N., Asymptotic Methods of the Statistic Analysis of the Time Series, BGU, Minsk 1999.

[4] Daubechies I., Ten Lectures on Wavelets, Scientific-Research Centre "Regular and chaotic dynamic", Izhevsk 2001.

[5] Dahlhaus R., Statistical methods in spectral estimation, Stochastische Mathematische Modelle, Heidelberg, December 12, 1992.

[6] Harris F.J., Using of the data tapers in harmonic analysis via method of discrete Fourier transformation, TSIER 1978, 66, 1, 60-96.

[7] Semenchuk N., On application of the spectral density estimate obtained via wavelets of stationary random process, $4^{\text {th }}$ International Workshop Computer Algebra Systems in Teaching and Research, Siedlce, Poland, January 31 - February 3, 2007, 281-286.

[8] Semenchuk N., Deytseva A., On Quadrature Formulas for Calculation of Approximation Wavelet Coefficients, Computer Algebra Systems in Teaching and Research, Mathematical Modeling in Physics, Civil Engineering, Economics and Finance, Siedlce 2011, 36-41.

[9] Zhurbenko I.G., Spectral Analysis of the Time Series, MGU Publishers, Moscow 1982.

[10] Zhurbenko I.G., Stochastic Process Simulation, MGU Publishers, Moscow 1990. 
